Ann. Sci. forest., 1971, 28 (3), 341-353.

\title{
CAUSES DE LA DÉFORMATION DES JEUNES DOUGLAS DANS LE LIMOUSIN
}

\author{
M. BONNEAU \\ avec la collaboration technique de A. Clément et Françoise Georges \\ Station de Recherches sur les Sols forestiers et la Fertilisation, \\ Centre national de Recherches forestières, I.N.R.A., \\ 54 - Nancy
}

RÉSUMÉ

Des déformations très graves atteignent les jeunes plantations de Douglas les plus vigoureuses dans l'ouest du Massif Central: les jeunes arbres prennent un port pleureur ou buissonnant. Des analyses foliaires ont montré que la nutrition en éléments majeurs ainsi qu'en fer et manganèse était satisfaisante.

L'élément responsable de la déformation est le cuivre dont la teneur est toujours inférieure au seuil de carence dans les aiguilles des arbres malades. La différence de nutrition entre les arbres, droits ou déformés, des zones atteintes et ceux des zones indemnes est hautement significative. Nos résultats concordent avec ceux de VAN Goor et de OldENCAMP.

L'épandage de $15 \mathrm{~g}$ de sulfate de cuivre par pied et une taille convenable permettent de redonner une forme normale aux jeunes Douglas déformés.

Il semble qu'il y ait aussi insuffisance de nutrition en zinc.

\section{INTRODUCTION}

Dans une courte note (Bonneau, 1969), nous avons signalé les fréquentes déformations survenant dans les plantations de Douglas du Limousin. A un âge qui peut varier de 2 à 5 ans la pousse principale se tord en $S$ ou se retourne complètement vers la terre ; la partie terminale, dans ce dernier cas, meurt et prend une teinte rouge. Une pousse latérale tente alors de prendre le relais, mais se déforme également, si bien que le jeune Douglas prend un port rampant, pleureur ou buissonnant (figures 1, 2 et 3). Dans quelques cas très rares, nous avons remarqué des pousses restées droites, mais mortes et rougies. Dans la plupart des cas, les arbres restent parfaitement vigoureux, 
leurs aiguilles sont grandes, vertes et saines, sans signe de chlorose ou de nécrose. Par contre, on note au niveau de la partie courbe de la tige, un aplatissement de celle-ci, accompagné très souvent d'une nécrose de la face inférieure.

Le port pleureur et la mortalité de la pousse ont été décrits par plusieurs auteurs (VAN Goor et al., 1966, Oldencamp et al., 1966) comme des symptômes de carence en cuivre et le seuil de carence a été évalué à 4,5 ppm environ par rapport à la matière sèche des aiguilles.

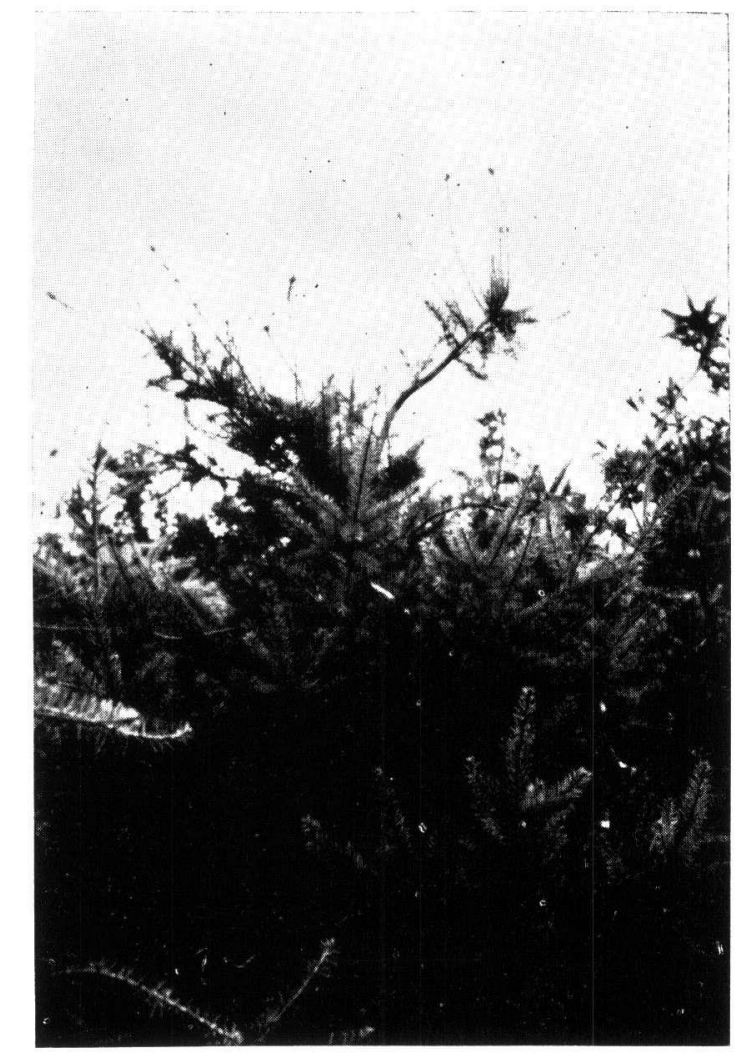

FIG. 1. - Douglas de 5 ans, moyennement déformé (Sornac, Corrèze)

FIG. 1. - Young Douglas-Fir, 5 years old, with moderate deformation (Sornac, Corrèze)

Dans le cas des plantations du Limousin la cause des déformations constatées pouvait donc être a priori attribuée à une carence en cuivre, d'autant plus que les carences en d'autres éléments donnent des symptômes différents, sauf, semble-t-il, la carence en zinc dans certains cas (STONE, 1966). Nous avons tenu, cependant, à assurer ce diagnostic par des analyses foliaires de plusieurs plantations. De plus, nous avons fourni des exemplaires de tige courbée et nécrosée au laboratoire de Pathologie du C.N.R.F. : notre collègue L. LANIER, n'y a relevé aucun champignon parasite. 


\section{1. — Les Plantations Étudiées}

A l'automne 1969, des prélèvements foliaires ont été effectués dans les plantations suivantes atteintes de déformations (Valeoux, Béchadergue: blocs 6 et 7 et Razès) ou non (Peyrilhac, Béchadergue: bloc 14).

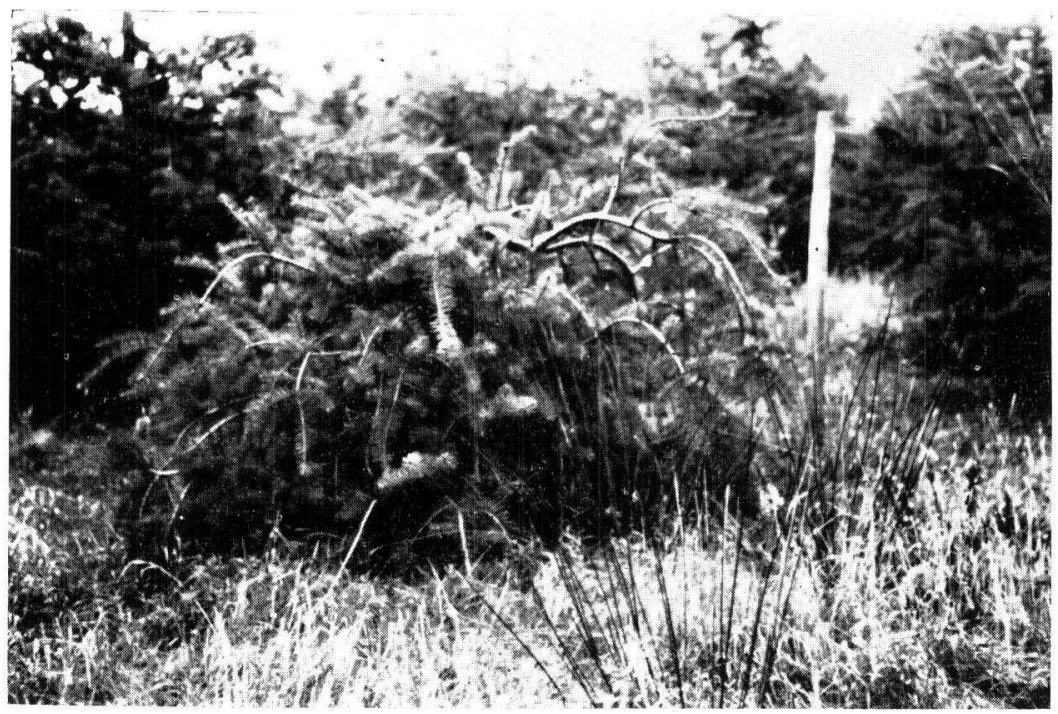

FIG. 2. - Douglas de 6 ans très déformé (Béchadergue, Hte-Vienne)

FIG. 2. - 6 years old Douglas-Fir, with heavy deformation

\section{1. - Plantation de Valeoux}

(Propriété de M. Duveir, Commune de la Nouaille, Creuse)

Ce jeune peuplement de 6 ans, installé sur une lande à fougère, sur granite d'Egletons (à biotite), est très vigoureux, mais beaucoup d'arbres sont déformés plus ou moins gravement. Nous avons prélevé des aiguilles des pousses de l'année des verticilles supérieurs sur 10 arbres droits et 10 arbres courbés. Nous n'avons effectué aucun prélèvement sur les arbres les plus déformés qui sont complètement rampants et disparaissent sous les fougères et qui sont concentrés sur un emplacement de quelques ares autour d'une petite dépression argileuse et mouilleuse.

\section{2. - Plantation comparative de provenances de Béchadergue} (Commune de Saint-Julien-le-Petit, Haute-Vienne)

Elle a été effectuée en 1965 par la Station d'Amélioration du C.N.R.F., dans une propriété comprenant à l'origine des terres cultivées et quelques îlots boisés, sur granite à deux micas. Dans une partie de cette plantation, à l'un des emplacements autrefois boisés, beaucoup d'arbres sont sévèrement atteints, avec un port complètement buissonnant, quelle que soit la provenance. Dans deux parcelles unitaires, l'une occu- 
pée par la provenance Nanaïmo (en abrégé NANA), bloc 6, l'autre par la provenance Molalla (en abrégé MOLA), bloc 7, 5 arbres sains et 5 arbres atteints ont été l'objet de prélèvements d'aiguilles.

Dans une autre partie de la plantation, sur un plateau autrefois cultivé, les mêmes provenances restent parfaitement droites malgré une croissance rapide. Nous avons prélevé les aiguilles de 5 arbres dans la parcelle occupée par la provenance NANA, bloc 14.

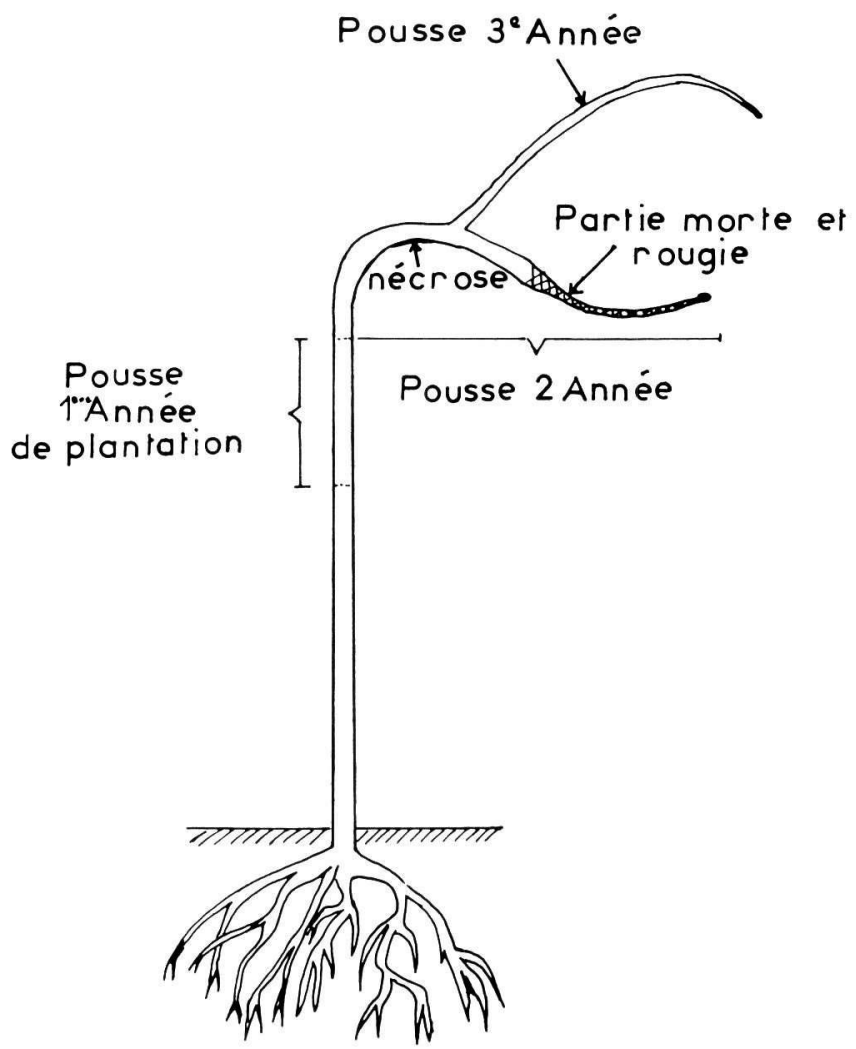

Fig. 3. - Schéma de la déformation d'un jeune Douglas

Fig. 3. - Figure of deformation of a young Douglas-Fir

\section{3. - Essai de fertilisation de Peyrilhac (Haute-Vienne)}

Il a été planté par l'A.F.O.C.E.L. *, en 1968, dans une ancienne parcelle cultivée, sur granite à biotite. Ces jeunes arbres, malgré une vitesse de croissance extrêmement spectaculaire (pousses de l'ordre de $1 \mathrm{~m}$ dès la deuxième année) restent parfaitement droits. Nous avons prélevé des aiguilles sur 5 arbres des parcelles non fertilisées.

* Nous remercions vivement M. de Champs, Ingénieur de cette société, de la visite qu'il nous a fait faire du dispositif et de l'autorisation qu'il nous a donnée d'y prélever des échantillons. 


\section{4. - Essai de fertilisation de Razès (Haute-Vienne)}

Il a été installé par nos soins dans un contrat du Fond Forestier National. Avant la plantation le sol, développé sur granite à deux micas et fortement humifère, était couvert d'une épaisse lande à callune. Plusieurs traitements sont comparés dans cet essai, les engrais étant appliqués en doses concentrées autour de chaque plant:

- $\mathrm{T}$ (non fertilisé).

- Ca.

- Ca P.

- Ca P K.

- Ca P K N.

La plantation a été effectuée au début de 1967 et la fertilisation eut rapidement des effets positifs; mais, dès la fin de 1968, de nombreuses déformations apparurent dans les traitements $\mathrm{Ca} P$ et surtout $\mathrm{Ca} P \mathrm{~K}$ et $\mathrm{Ca} P \mathrm{PN}$ où les plants manifestaient la croissance la plus rapide, tandis qu'ils restaient droits dans les modalités Ca et $\mathrm{T}$. A la fin de 1969, nous avons prélevé des aiguilles de 5 arbres dans chacune des catégories suivantes :

Bloc 8: témoins sains - $\mathrm{Ca} P$ sains - $\mathrm{Ca} \mathrm{P} \mathrm{KN}$ sains - $\mathrm{Ca} \mathrm{PKN}$ déformés. Bloc 7: Ca P K N sains - Ca P K N déformés - Ca P K N très déformés.

Contrairement aux plantations précédentes où les prélèvements effectués sur chaque arbre ont été analysés individuellement, les aiguilles des 5 arbres de chacune des catégories prélevées à Razès ont été mélangées avant analyse pour constituer un échantillon moyen.

\section{2. - LES ANALYSES ET LEUR INTERPRÉTATION}

Les analyses suivantes ont été pratiquées après séchage des échantillons à $65^{\circ}$. génée ;

- $\mathrm{N}, \mathrm{P}_{2} \mathrm{O}_{5}, \mathrm{~K}, \mathrm{Ca}, \mathrm{Mg}$ après minéralisation dans l'acide sulfurique et l'eau oxy-

- $\mathrm{Mn}, \mathrm{Fe}, \mathrm{Zn}, \mathrm{Cu}$ après minéralisation au four à moufle, reprise à $\mathrm{ClH}$ et séparation des éléments sur résine échangeuse de cations (technique mise au point par A. Clément) ;

- B après minéralisation au four et reprise par l'acide acétique (dosage à la 1-1'-dianthrimide).

Les résultats analytiques (moyennes des analyses individuelles pour Valeoux, Béchadergue, Peyrilhac et analyse des échantillons moyens pour Razès) sont donnés dans le tableau 1.

On peut constater que les éléments majeurs $\mathrm{N}, \mathrm{P}_{2} \mathrm{O}_{5}, \mathrm{~K}, \mathrm{Ca}, \mathrm{Mg}$ sont en concentration suffisante dans les aiguilles et qu'un manque de nutrition dans ce domaine ne peut en aucun cas expliquer les désordres de croissance constatés. Les arbres déformés ne sont pas notablement moins riches en éléments nutritifs que les arbres sains. Nous pouvons cependant constater au passage la forte concentration en azote des Douglas de Peyrilhac qui ont, de loin, parmi tous ceux soumis à l'analyse, la plus forte croissance. 
TABLEAU 1 - TABLE 1

Concentration des divers éléments analysés dans les aigu:lles

Concentration of elements in needles

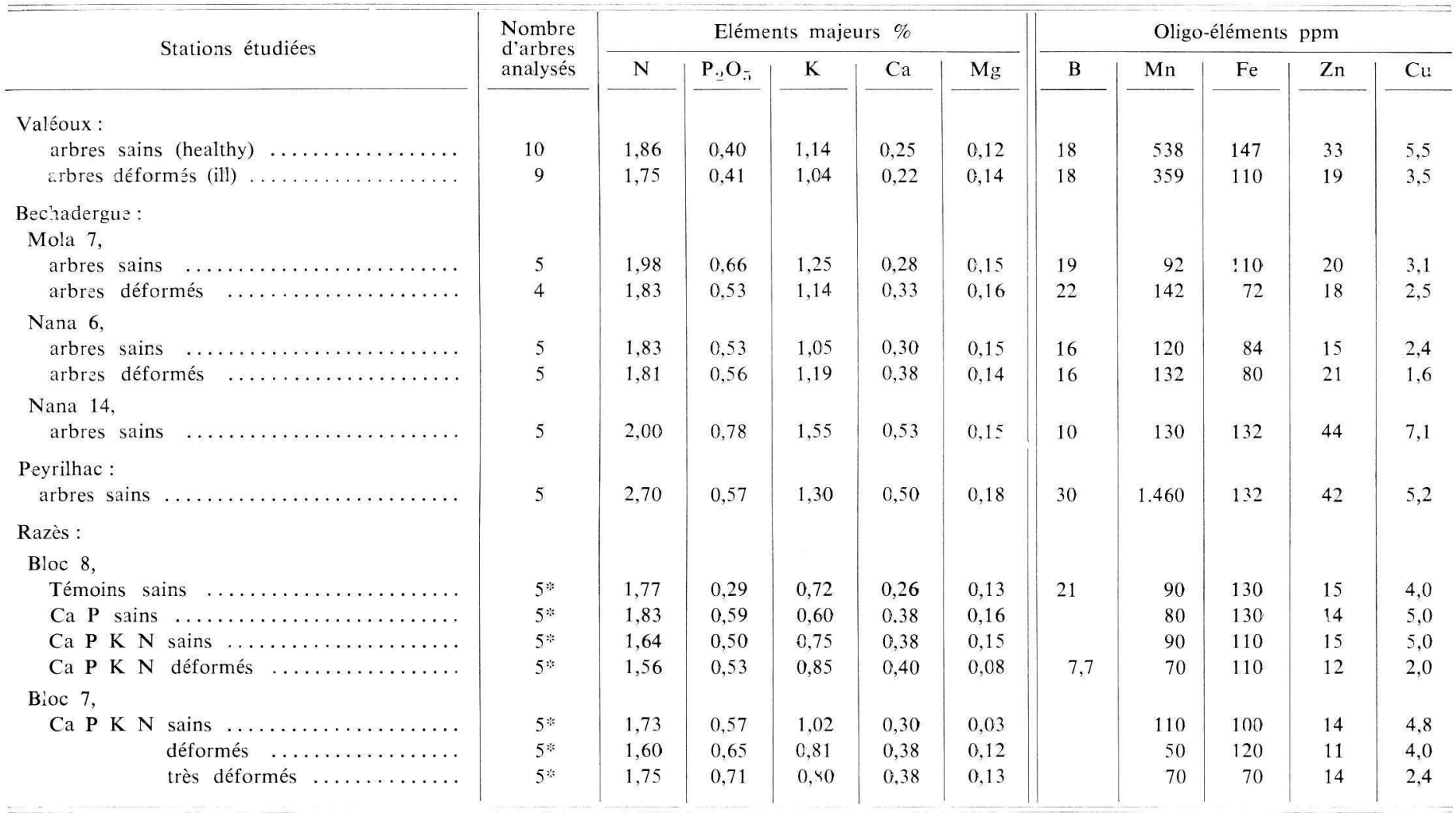

N.B. - Lorsque le nombre d'arbres analysés est suivi du sigñ *, cela indique que l'analyse a porté sur le mélange des différenis échantilions. 
Nous avons donc accordé le maximum d'attention aux oligo-éléments. Plusieurs comparaisons sont théoriquement possibles à partir de l'échantillonnage effectué :

a) Comparaison des concentrations en oligo-éléments des arbres sains et des arbres déformés dans la même station :

- Valeoux sains/Valeoux déformés,

- Nana 6 sains/Nana 6 déformés,

- Mola 7 sains/Mola 7 déformés.

b) Comparaison de l'ensemble des arbres sains aux arbres déformés pour l'ensemble des stations où les symptômes se manifestent :

- (Valeoux + Nana 6 + Mola 7) sains/(Valeoux + Nana $6+$ Mola 7) déformés.

c) Comparaison d'arbres malades aux arbres de stations où la déformation n'apparaît pas :

- Nana 14 sains/Nana 6 déformés,

- Peyrilhac sains/Nana 6 déformés.

d) Comparaison de tous les arbres sains et déformés, de l'ensemble des stations atteintes, aux arbres des stations indemnes :

- (Valeoux + Mola $7+$ Nana 6)/Peyrilhac + Nana 14.

En réalité, toutes les comparaisons possibles n'ont pas été effectuées car il s'est avéré, dans beaucoup de cas, que la condition préalable d'égalité des variances des deux populations à comparer n'était pas remplie. Si nous avions prélevé davantage d'arbres dans chaque station, nous aurions sans doute évité certains de ces écueils, mais l'analyse par voie chimique des oligo-éléments est un long travail que nous ne pouvions multiplier à loisir. Le tableau 2 donne, pour chaque oligo-élément et pour chaque comparaison valable, la valeur du test $t$ et le degré de signification.

\section{1. - Le bore}

Cet élément ne fournit aucune explication puisqu'aucune des comparaisons n'est significative. Le seuil de carence admis pour le bore, $10 \mathrm{ppm}$ environ, n'est d'ailleurs atteint dans aucune plantation, sauf peut-être dans le traitement $\mathrm{Ca} P \mathrm{P} \mathrm{N}$ de Razès (tableau 1). Mais il s'agit simplement d'un phénomène local auquel on ne peut donner aucune portée générale.

\section{2. - Le manganèse}

Deux comparaisons seulement sont valables et ce ne sont pas les plus intéressantes. Dans l'un des cas (Valeoux) les arbres déformés sont plus pauvres en manganèse, dans l'autre (Mola 7) ils sont plus riches. On ne peut donc pas mettre en cause cet élément, sa concentration dans les aiguilles étant de plus partout très largement supérieure au seuil de carence reconnu pour les résineux : 20 à $40 \mathrm{ppm}$. On voit d'ailleurs mal pourquoi, sur ces sols acides développés sur roches-mères cristallines généralement riches en biotite, l'alimentation en manganèse laisserait à désirer. 
TABLEAU 2 - TABLE 2

Comparaisons des concentrations de divers oligo-éléments dans les aiguilles de un an de Douglas sains et de Douglas déformés

Comparison of trace-elements concentrations in one-year needles of healthy or ill Douglas-fir

\begin{tabular}{|c|c|c|c|c|c|c|}
\hline & \multirow{3}{*}{$\begin{array}{c}\begin{array}{c}\text { Nombre } \\
\text { de ddl }\end{array} \\
17\end{array}$} & \multicolumn{5}{|c|}{ Valeurs de $t$ et degré de signification pour les éléments étudiés } \\
\hline & & B & $\mathrm{Mn}$ & $\mathrm{Fe}$ & $\mathrm{Zn}$ & $\mathrm{Cu}$ \\
\hline Valéoux sains/Valéoux déformés $\ldots \ldots \ldots \ldots \ldots$ & & $\begin{array}{c}0,221 \\
\text { NS }\end{array}$ & $\begin{array}{c}2,25 \\
+\end{array}$ & $\begin{array}{r}3,70 \\
++\end{array}$ & & \\
\hline Nana 6 sains/Nana 6 déformés $\ldots \ldots \ldots \ldots \ldots$ & 8 & $\begin{array}{r}0,88 \\
\text { NS }\end{array}$ & & NS & $\begin{array}{l}3,05 \\
--\end{array}$ & $\begin{array}{c}0,88 \\
\text { NS }\end{array}$ \\
\hline Mola 7 sains $/$ Mola 7 déformés $\ldots \ldots \ldots \ldots \ldots$ & 7 & $\begin{array}{c}1,097 \\
\text { NS }\end{array}$ & $\begin{array}{l}3,36 \\
-\end{array}$ & $\begin{array}{r}2,71 \\
+\end{array}$ & & \\
\hline Nana 14 sains/Nana 6 déformés $\ldots \ldots \ldots \ldots \ldots$ & 8 & $\begin{array}{r}2,22 \\
\mathrm{NS}\end{array}$ & & $\begin{array}{c}7,14 \\
+++\end{array}$ & & $\begin{array}{c}5,24 \\
+++\end{array}$ \\
\hline Peyrilhac sains/Nana 6 déformés $\ldots \ldots \ldots \ldots \ldots$ & 8 & & & $\begin{array}{c}4,54 \\
++\end{array}$ & & $\begin{array}{r}3,72 \\
++\end{array}$ \\
\hline $\begin{array}{l}\text { (Nana } 6+\text { Mola } 7+\text { Valéoux }) \text { sains } /(\text { Nana } 6+ \\
\text { Mola } 7+\text { Valéoux }) \text { déformés } \ldots \ldots \ldots \ldots \ldots \ldots\end{array}$ & 36 & $\begin{array}{r}0,71 \\
\text { NS }\end{array}$ & & $\begin{array}{l}3,49 \\
++\end{array}$ & & $\begin{array}{r}2,78 \\
++\end{array}$ \\
\hline $\begin{array}{l}\text { (Peyrilhac }+ \text { Nana } 14) \text { sains } /(\text { Nana } 6+\text { Mola } 7+ \\
\text { Valéoux) (sains }+ \text { déformés) } \ldots \ldots \ldots \ldots \ldots \ldots \ldots\end{array}$ & 46 & & & $\begin{array}{r}2,16 \\
+\end{array}$ & $\begin{array}{c}6,4 \\
+++\end{array}$ & $\begin{array}{c}4,43 \\
+++\end{array}$ \\
\hline
\end{tabular}

Degré de signification : Si l'élément considéré est plus concentré dans les arbres sains :,,++++++ à $1 \%, 1 \%, 5 \%$ respectivement. Si l'élément considéré est moins concentré dans les arbres sains: - - , - , - à $1 \%, 1 \%, 5 \%$.

Degree of signification: if the element is more concentrated in healthy trees:,,++++++ at $1 \%, 1 \%, 5 \%$ level if the element is less concentrated in healthy trees: - - ... - at 1 , $, 1 \%, 5 \%$ level. 


$$
\text { 2.3. - Le fer }
$$

Toutes les comparaisons sont valables et montrent toutes, sauf une, une différence significative entre arbres sains et arbres déformés, que ce soit à l'intérieur des stations où se manifestent les désordres ou entre stations atteintes et stations indemnes.

Il ne nous paraît cependant pas possible de charger le fer de la responsabilité des désordres constatés car :

- les teneurs dépassent le seuil de carence admis jusqu'à nouvel ordre (bien que mal connu) de 25 à 40 ppm,

- la chlorose caractéristique de la carence en fer n'a été constatée nulle part,

- les conditions édaphiques déjà exposées pour le manganèse rendent invraisemblable une insuffisance d'alimentation en fer.

$$
\text { 2.4. - Le zinc }
$$

Les comparaisons entre plants sains et plants déformés d'une même station sont, ou bien non valables, ou bien non significatives, ou bien en faveur des plants malades. Mais la comparaison globale des stations exemptes de déformations et des stations atteintes est très hautement significative et montre un niveau de zinc supérieur dans les populations saines.

Le seuil de carence en zinc se situe aux environs de $10 \mathrm{ppm}$ pour Pinus radiata; celui du Douglas étant inconnu, il n’est pas impossible que les teneurs relevées dans les stations atteintes (12 ppm à Razès à 21 ppm à Béchadergue Nana 6) en soient proches, alors que les plantations saines ont des teneurs très supérieures: plus de $40 \mathrm{ppm}$ à Peyrilhac et à Béchadergue Nana 14.

On ne peut donc éliminer complètement l'hypothèse d'une carence en zinc.

\section{5. - Le cuivre}

Les comparaisons à l'intérieur d'une station où existent des arbres déformés sont non valables ou non significatives, mais pour l'ensemble des zones atteintes les arbres indemnes sont, de manière très significative, plus riches en cuivre que les arbres malades. De même, les tests de comparaison entre les arbres de zones indemnes et les arbres déformés de stations atteintes sont très significatifs: (Nana 6 et Peyrilhac), ainsi que la comparaison entre les Douglas courbés ou non des zones atteintes et ceux des stations saines.

De plus, les concentrations constatées chez les arbres déformés sont toujours inférieures au seuil de carence de $4,5 \mathrm{ppm}$ admis pour le Douglas. Il en est souvent de même chez les arbres sains d'une station où sévit la maladie. A l'intérieur de ces stations, la teneur en cuivre est, quoique de manière non significative sur le plan statistique, plus faible dans les arbres déformés. Enfin à Razès, on constate que la concentration en cuivre est de plus en plus faible lorsqu'on va des arbres droits peu fertilisés aux arbres déformés et très déformés qui ont bénéficié d’un apport très complet d’engrais. 


\section{3. - CONCLUSION ET DISCUSSION}

De cette étude par analyse foliaire des causes de déformations des jeunes Douglas, nous avons conclu à une excellente nutrition en éléments majeurs, bien que l'optimum en azote ne semble pas toujours atteint. Nous avons admis l'éventualité d'une insuffisance d'alimentation en zinc et la possibilité d'une carence en bore ; mais cette dernière n'existerait que dans une seule des stations étudiées, n'apportant donc pas une explication générale au problème.

Le cuivre est le seul oligo-élément pour lequel on trouve à la fois une différence de nutrition entre les zones atteintes par la déformation et les zones indemnes, une différence de concentration entre arbres sains et malades danı l'ensemble des stations atteintes, et une concentration dans les aiguilles des arbres déformés nettement inférieure au seuil de carence. On ne doit pas s'étonner qu'à l'intérieur d'une station la différence ne soit pas toujours significative entre les Douglas droits et les Douglas buissonnants ou courbés : en effet ces stations sont homogènes, tous les arbres disposent d'un espace suffisant (il n'y a dans le jeune âge ni dominants ni dominés) et on ne voit pas pourquoi le niveau de nutrition serait différent des uns aux autres. Que certains soient atteints et d'autres indemnes signifie seulement que pour l'ensemble de la population les conditions d'alimentation sont telles que l'on se trouve aux limites du domaine de carence, celle-ci pouvant apparaitre ou non suivant les microvariations du sol ou les degrés de sensibilité individuelle. Il faut atteindre une grande précision dans l'analyse, en comparant un grand nombre d'arbres, pour donner une valeur statistique aux petites différences qui peuvent exister.

Nos conclusions confirment donc celles des auteurs étrangers (VAN Goor, OldenCAMP et al.) et permettent bien de faire correspondre aux mêmes symptômes la même cause.

Un essai de traitement curatif direct apporte d'ailleurs une nouvelle confirmation. En 1969, nous avons épandu à Razès $15 \mathrm{~g}$ de sulfate de cuivre (dont $5 \mathrm{~g}$ au printemps et $10 \mathrm{~g}$ à l'automne) au pied de chaque Douglas des traitements $\mathrm{CaP}, \mathrm{CaPK}$ et CaPKN, puis à la fin de l'hiver 1969-1970, nous avons coupé les arbres déformés juste au-dessous de la courbure. Les nouvelles pousses de 1970, parfois multiples, sont parfaitement droites comme on peut le voir sur la figure 4 prise à l'automne 1970 et où l'on distingue également, remise en place pour les besoins de la photo, la partie déformée qui avait été coupée au printemps.

Dans cette reconstitution des arbres après épandage de sulfate de cuivre et taille, on peut distinguer schématiquement deux cas :

a) La déformation s'était produite sur la dernière pousse formée, celle de 1969 (ce qui indiquait d'ailleurs que la dose de $5 \mathrm{~g}$ de sulfate de cuivre épandue au printemps avait été trop faible ou administrée trop tardivement): la taille a laissé subsister la partie inférieure de cette pousse restée droite et portant plusieurs bourgeons vigoureux. Plusieurs de ces bourgeons se sont développés en nouvelles pousses, droites et également vigoureuses. Une nouvelle taille ne laissant subsister qu'une de ces pousses est donc nécessaire. 
b) La déformation s'était produite plus précocement, sur une pousse de 1968 généralement, parfois de 1967. Au moment de la taille, cette pousse avait déjà $2 \mathrm{~cm}$ de diamètre, parfois plus, et la partie droite qui subsistait après la taille ne portait plus aucun bourgeon, tous s'étant développés en rameaux latéraux au cours de l'année 1969. Ces rameaux latéraux de base de pousse étaient d'ailleurs souvent assez peu vigoureux, et il n'était pas du tout certain, a priori, que l'arbre taillé réussirait à refaire une flèche.

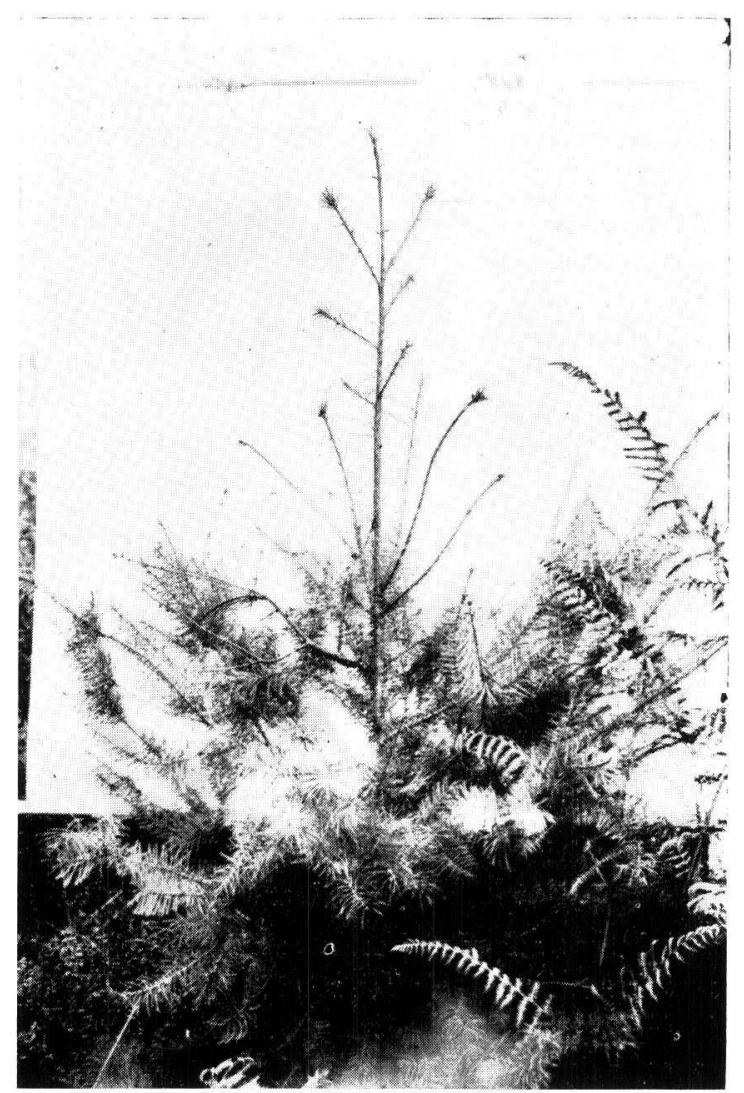

FIG. 4. - Jeune Douglas de 4 ans après apport de sulfate de cuivre et taille. En bas et à gauche de la nouvelle pousse: ancienne tige déformée et éliminée par la taille

FIG. 4. - 4 years old Douglas-Fir, after speading of copper sulfate and pruning. Below, on the lett of the new shoot: deformated shoot of the last year, removed by pruning

En fait, tous y sont parvenus sans difficulté par redressement d'un ou plusieurs rameaux latéraux et développement extrêmement vigoureux de leur bourgeon terminal en une flèche normale. La figure 4 montre assez nettement, un peu au-dessous de la branche latérale coupée et remise en place pour la photo, la crosse formée à l'endroit où le rameau s'est redressé, puis, dépassant nettement la branche coupée, l'ancien rameau redressé, assez gros et à écorce d'aspect rugueux, et enfin la nouvelle pousse de 1970 beaucoup plus longue, plus fine, à aspect plus lisse et sur laquelle une pousse d'août a 
amené le développement de nouveaux rameaux latéraux (les mains qui tiennent l'écran donnent l'échelle). Une nouvelle taille est nécessaire, comme dans le cas précédent, si plusieurs flèches se sont ainsi formées.

En ce qui concerne le déclenchement des déformations, on pourrait penser qu'il s'agit d'un déséquilibre entre éléments majeurs et le cuivre. Le fait que, dans la plantation de Razès, ce soit dans les parcelles les plus fertilisées (traitements CaPN et CaPKN) que l'on ait rencontré presque toutes les déformations conforte ce point de vue.

En réalité, nous pensons que la réalité est plus complexe. En effet, les arbres sains, dans les stations atteintes, sont un peu plus riches en azote, phosphore et potassium que les arbres déformés. Surtout l'excellente plantation de Peyrilhac, avec son taux très élevé d'azote et de potassium, le placeau Nana 14 de Béchardergue où les arbres, riches en azote, phosphore et potassium, ne le cèdent pas en vigueur aux meilleurs arbres droits des placeaux Nana 6 et Mola 7, montrent qu'on peut avoir des Douglas à la fois vigoureux, largement alimentés en éléments majeurs et droits.

Il semble donc qu'une croissance rapide n'entraîne la carence en cuivre, un déséquilibre entre cet oligo-élément et les éléments majeurs, et finalement la déformation que dans des circonstances édaphiques particulières. Ces circonstances ne sont pas élucidées à l'heure actuelle et nous avons simplement remarqué :

- que les stations indemnes Nana 14 et Peyrilhac sont d'anciens sols cultivés assez pauvres en matière organique et peut-être enrichis en cuivre par des applications de produits anticryptogamiques;

- que la différence relevée en faveur des stations saines pour le cuivre et le zinc existe aussi pour le fer. Ce sont donc plusieurs oligo-éléments métalliques lourds qui semblent moins abondamment assimilés dans les sols d'anciennes forêts ou de landes riches en humus, et il est logique de faire l'hypothèse d'une fixation énergique de ces cations par la matière organique. Des recherches ultérieures chercheront à vérifier ce point de vue.

Par ailleurs, une expérience est déjà entreprise à Razès pour savoir quelle dose de cuivre il faudrait appliquer en complément des engrais classiques pour éviter l'apparition des déformations.

Reçu pour publication en mai 1971.

\section{SUMMARY}

\section{CAUSES OF DEFORMATIONS OF THE YOUNG DOUGLAS-FIRS IN LIMOUSIN}

The most vigourous of the Young Douglas-Fir plantations in West of Massif Central suffer from heavy deformations: the shoots of the young trees are curved in $\mathrm{S}$, or the trees have a weeping or bushy appearance.

Foliar diagnosis showed that the nutrition in major elements, manganese and iron is good. The responsible element of that deformation is copper, the concentration of which in the one-year needles of ill trees is always below the deficiency levei. Difference between nutrition of ill or healthy trees of sites where the deformation appears and nutrition on normal sites is highly significant. Our own conclusions agree well with those of VAN GooR and OLdencamp.

Broadcast spreading of $15 \mathrm{~g}$ copper sulfate to individual trees and a convenient pruning permitt to restore a normal shape. 


\section{ZUSAMMENFASSUNG}

\section{URSACHEN DER MISSGESTALTUNGEN DER JUNGEN DOUGLASIEN IM LIMOUSIN}

Bestwüchsige junge Douglasienaufforstungen im Westen des Zentralmassiv's leiden an schweren Missgestaltungen. Die Pflanzen haben eine buschige Wuchsform und herabhängende Zweige. Die Nadellanalysen zeigten einen guten Ernährungszustand an Makroelementen sowie Eisen und Mangan.

Bei den kranken Bäumen konnte eine Karrenz an Kupfer festgestellt werden, welche die Missgestaltung hervorruft.

Die Unterschiede im Kupfergehalt zwischen Standorten mit und ohne Krankheitserscheinungen sind hoch signifikant. Die Unterschiede zwischen gesunden und kranken Pflanzen eines kranken Standortes sind jedoch nicht signifikant. Unsere Ergebnisse stimmen mit jenen von Van Goor und von OlNenCAMP gut überein.

Die Ausstreuung von $15 \mathrm{~g}$ Kupfer-sulfat um jede Pflanze und ein entsprechender Schnitt geben den erkrankten Pflanzen wiederum eine normale Form. Desweiteren scheint auch eine Karrenz an Zink zu bestehen.

\section{RÉFÉRENCES BIBLIOGRAPHIQUES}

Bonneau M. Déformations de jeunes Douglas dans le Massif Central, R.F.F., $\mathrm{n}^{\circ}$ 1, 1970.

Oldencamp L., Smilde K. W. Copner defiiciency in Douglas-fir (Pseudotsuga menziesii). Plant and Soil, 25, 1966.

Stoate T. N. Nutrition on the pine, Forestry and timber bureau (Australia), $\mathrm{n}^{\circ}$ 30, 1950.

StONE E. L. Microelement nutrition of forest trees: a review in «Forest fertilization, theory and practice». Symposium of forest fertilization, Gainesville, Tennessee Valley authority, 1968.

Van Goor C. P., Henkens C. H. Groeimisvormingen bij Douglas en Fijnspar en Sporenelementen (Netherlands). Bosbouwpwefstation Korte Medd., 76, 1966. 